\title{
Auto-Regulação da Aprendizagem em Crianças e Adolescentes Filhos de Vítimas de Violência Doméstica Contra Mulheres
}

\author{
Self-Regulation of Learning in Children and Adolescents whose Mothers \\ were Victims of Domestic Violence
}

\author{
Micaela Preto \& Paulo A. S. Moreira* \\ Universidade Lusíada do Porto, Porto, Portugal
}

\begin{abstract}
Resumo
A violência doméstica contra mulheres afecta negativamente o desenvolvimento de alguns domínios de auto-regulação. Contudo, pouco se conhece sobre o seu impacto na auto-regulação da aprendizagem. Este estudo avaliou a auto-regulação da aprendizagem em 50 fillhos de vítimas de violência doméstica contra mulheres. O grupo de filhos de vítimas apresentou níveis inferiores de rendimento académico. O grupo de filhos de vítimas simultaneamente de violência psicológica e física registou níveis inferiores nas dimensões de Avaliação e Execução de auto-regulação da aprendizagem. Estes resultados suportam as evidências de que a exposição à violência doméstica afecta negativamente o desenvolvimento da auto-regulação. Para além disso, sugerem que a exposição a violência familiar afecta negativamente também algumas dimensões da auto-regulação da aprendizagem.

Palavras-chave: Violência na família, auto-regulação, aprendizagem, aprendizagem auto-regulada.
\end{abstract}

\begin{abstract}
Domestic violence against women has a negative impact on several domains of self-regulation. However, very little is known about its impact on self-regulated learning. This study evaluated self-regulation of learning in children and adolescents exposed to domestic violence. Fifty children and adolescents, whose mothers were victims of domestic violence and 50 whose mothers were not, participated in the study. The group of children and adolescents whose mothers were victims of intimate partner violence registered lower academic performance and the group exposed simultaneously to psychological and physical violence registered lower scores on the self-regulation dimensions of evaluation and execution. The results support the evidence that exposure to domestic violence has a negative impact on self-regulation. Also, they suggest that exposure to domestic violence has a negative impact in some dimensions of self-regulated learning.

Keywords: Domestic violence, self-regulation, learning, self-regulated learning.
\end{abstract}

\section{Auto-Regulação da Aprendizagem em Filhos de Vítimas de Violência Doméstica}

Os maus-tratos a crianças podem tomar diversas formas (Higgins \& McCabe, 2001), incluindo o abuso sexual, abuso físico, abuso psicológico, e negligência. As diversas tipologias de maus-tratos (e.g., Higgins, 2004; McGee \& Wolfe, 1991; Wolfe \& McGee, 1994) consideram como vítimas tanto os indivíduos a que o abuso é dirigido (por exemplo, a esposa que sofre algum destes tipos de abuso), como crianças e adolescentes que testemunham a situações de abuso (por exemplo, filhos assistirem a violência entre os progenitores; Edwards, Holden,

\footnotetext{
* Endereço para correspondência: Centro de Investigação em Psicologia para o Desenvolvimento, Universidade Lusíada do Porto, Rua Doutor Lopo de Carvalho, Porto, Portugal 4369-006. E-mail: paulomoreira@por.ulusiada.pt
}

Felitti, \& Anda, 2003; Higgins, 2004; Higgins \& McCabe, 2000). Assistir a violência de um progenitor ao outro e ser alvo de violência dirigida a si, são fenómenos que aparecem frequentemente associados (Coohey, 2004; T. I. Herrenkohl, Sousa, Tajima, R. C. Herrenkohl, \& Moylan, 2008; Kitzmann, Gaylord, Holt, \& Kenny, 2003). Assim, grande parte dos estudos com filhos de vítimas de violência doméstica contra mulheres considera a co-ocorrência dos dois tipos de abuso (ser vítima directa de violência e testemunhar violência entre os progenitores, Lepisto, Luukkaala, \& Paavilainen, 2011), com o objectivo de garantir maior validade ecológica. Este é também o caso deste estudo, no qual os filhos de vítimas foram considerados tanto como vítimas directas de violência quanto testemunhas da violência entre os progenitores.

Existe um robusto corpo de estudos que demonstra que a exposição à violência doméstica contra mulheres afecta negativamente múltiplos domínios de funcionamento nos filhos das vítimas (e.g., Noble, Tottenham, \& Casey, 2005; 
Stover \& Berkowitz, 2005; Wolfe, Crooks, Lee, McIntyreSmith, \& Jaffe, 2003) mesmo quando outros factores são controlados (Hazen, Connelly, Kelleher, Barth, \& Landsyerk, 2006; Jaffee, Moffitt, Caspi, Taylor, \& Aseneault, 2002), e independentemente da cultura (Vermeiren, Schwab-Stone, Deboutte, Leckman, \& Ruchkin, 2003). Filhos de vítimas de violência doméstica contra mulheres apresentam maior risco de apresentarem défices de competências sociais (Edleson \& Gewirtz, 2007; Karreman, Tujil, Aken, \& Dekovic, 2006; Margolin \& Gordis, 2000), emocionais (Hirigoyen, 1999; Kitzmann et al., 2003; Wolfe et al., 2003) de resolução de problemas (Graham-Bermann \& Levendosky, 1998) e dificuldades académicas (Halford, Sanders, \& Behrens, 2001; Noble et al., 2005). Os níveis elevados de reactividade comportamental associados (Margolin, 1998) causam prejuízo no desenvolvimento neurocognitivo (Margolin \& Gordis, 2000; Noble et al., 2005) e supressão ambiental do Q.I. (Koenen, Moffitt, Caspi, Taylor, \& Purcell, 2003).

Estes efeitos negativos têm sido explicados não apenas pelo facto de os filhos das vítimas assistirem à situação de abuso (Howard, Feigelman, Li, Cross, \& Rachuba, 2002), mas também pela perturbação que a violência doméstica contra mulheres introduz no funcionamento biopsicossocial das vítimas, o que afecta os seus comportamentos parentais e as suas interacções com os filhos. Como é descrito pelo modelo do perpetrador sequencial, os comportamentos das mulheres vítimas de violência doméstica para com os filhos (nomeadamente as práticas parentais) são significativamente afectados pelo abuso (Coohey, 2004). É frequente que os abusos perpetrados pelo abusador contra a vítima (cônjuge) co-existam em padrões semelhantes de abuso nos filhos (e.g., Coohey, 2004; T. I. Herrenkohl et al., 2008).

A auto-regulação da aprendizagem tem sido definida como as dinâmicas de organização de vários processos cognitivos (planeamento, execução e avaliação) e de gestão de elementos internos e externos com vista à prossecução de um objectivo, que permitem que o indivíduo assuma um papel activo na obtenção de uma resposta adaptativa aos desafios (Rosário, 2001), ajustando a intensidade da emoção ou do comportamento à situação (Conway, 2009; Gestsdóttir \& Lerner, 2008; Shonkoff \& Phillips, 2000). Mais do que em termos dicotómicos (presença versus ausência), a auto-regulação tem sido conceptualizada um continuum (Deci, Ryan, \& Williams, 1996; Ryan \& Deci, 2000) que prediz os níveis de ajustamento dos indivíduos, para além de moderar a relação entre múltiplos riscos e ajustamento, resiliência e vulnerabilidade (Lengua, 2002). Os mecanismos de autoregulação têm-se revelado determinantes na compreensão do comportamento humano em diversos domínios, incluindo na educação (Zimmerman \& Schunk, 2001), saúde (Bandura, 2005; Boekaerts, Maes, \& Karoly, 2005), funcionamento emocional (Gross, 1998, 2002; Ochsner \& Gross, 2005) e no desempenho de tarefas (Carver \& Scheir, 1982; Kanfer \& Karoly, 1972).
O processo auto-regulatório da aprendizagem está intimamente associado aos pensamentos, sentimentos e acções desenvolvidas e orientadas pelo aluno para a prossecução de objectivos pré-estabelecidos (Zimmerman \& Martinez-Pons, 1990), através da utilização sistemática de estratégias cognitivas, metacognitivas, motivadoras e comportamentais (Schunk, 1994; Zimmerman, 2000). Alunos auto-regulados tendem a ser caracterizados como decididos, estratégicos e persistentes no decurso da aprendizagem. São capazes de avaliar os seus progressos em relação a objectivos previamente definidos e aferir o seu comportamento de acordo com essas avaliações (Rosário, 2004; Rosário, Soares, Núñez Perez, GonzálezPienda, \& Rubio, 2004; Silva, Duarte, Sá, \& Simão, 2004; Zimmerman \& Schunk, 2001), e de utilizar estratégias específicas para alcançar objectivos escolares (Zimmerman, 1998), que incluem a organização e transformação dos conteúdos a serem aprendidos, a procura de informação, a repetição e a utilização da memória compreensiva (Zimmerman \& Martinez-Ponz, 1990).

O debate acerca de quais os modelos mais adequados para a compreensão das dinâmicas envolvidas em situações de abuso permanece em aberto, com alguns autores a salientarem as vantagens dos modelos de múltiplos riscos (e.g., Margolin \& Gordis, 2000; Masten \& Coatsworth, 1998) e outros a demonstrarem a maior adequabilidade dos modelos específicos (e.g., MartinezTorteya, Bogat, von Eye, \& Levendosky, 2009). A exposição dos indivíduos a factores de risco cumulativos está associada a um funcionamento mais pobre do que o de indivíduos com menos factores de risco (Jaffee, Caspi, Moffitt, Polo-Tomás, \& Taylor, 2007), aumentando a probabilidade de dificuldades acrescidas em múltiplos domínios de funcionamento, incluindo nas tarefas desenvolvimentais normativas.

Sendo que os filhos de vítimas de violência doméstica contra mulheres estão em maior risco para virem a apresentar dificuldades académicas (Kitzmann et al., 2003; Margolin \& Gordis, 2000), e sendo que a auto-regulação da aprendizagem está associada à qualidade dos resultados académicos (Zimmerman \& Schunk, 2001), a compreensão da auto-regulação da aprendizagem assume grande relevância para a compreensão das trajectórias de resiliência ou de vulnerabilidade desta população.

O objectivo deste estudo foi avaliar a auto-regulação da aprendizagem em filhos de vítimas de violência doméstica contra mulheres, testando a hipótese de este grupo apresentar níveis mais baixos nas dimensões da auto-regulação da aprendizagem.

\section{Método}

\section{Participantes}

Como pode ser consultado na Tabela 1, participaram neste estudo 100 crianças e adolescentes, dos quais 50 (50\%) eram fillhos de vítimas de violência doméstica contra mulheres e 50 (50\%) filhos de mulheres não vítimas 
de violência doméstica. Dos filhos de vítimas de violência doméstica 25 (50\%) eram género masculino e 25 $(50 \%)$ do género feminino, $5(10 \%)$ frequentavam o $1^{\circ}$ ciclo (ensino fundamental I), $44(88 \%) 3^{\circ}$ ciclo (ensino fundamental II) e 1 (2\%) o Secundário (ensino médio). Das mulheres vítimas de violência doméstica, 17 (34\%) tinham o $1^{\circ}$ ciclo (ensino fundamental I), $29(58 \%)$ o $3^{\circ}$ ciclo (ensino fundamental II), 2 (4\%) o Secundário (ensino médio) e 2 (4\%) Licenciatura (ensino superior).

Tabela 1

Caracterização da Amostra - Idade, Género e Escolaridade das Mulheres Vítimas e Não Vítimas de Violência Doméstica Contra Mulheres e dos Seus Filhos

\begin{tabular}{lccc}
\hline & $\begin{array}{c}\text { Total } \\
(N=100)\end{array}$ & $\begin{array}{c}\text { Filhos de vítimas } \\
(N=50)\end{array}$ & $\begin{array}{c}\text { Filhos de não vítimas } \\
(N=50)\end{array}$ \\
\hline Idade & & & \\
$\quad$ Minimo & 10 & 10 & 11 \\
Maximo & 16 & 16 & 15 \\
Idade $(D P)$ & $12,89(1,41)$ & $12,60(1,74)$ & $13,18(0,92)$ \\
Género & & & \\
Masculino & $43(42,6 \%)$ & $25(50 \%)$ & $18(36 \%)$ \\
Feminino & $57(56,4 \%)$ & $25(50 \%)$ & $32(64 \%)$ \\
Escolaridade dos filhos & & & \\
$1^{\circ}$ Ciclo (ensino fundamental I) & $5(5 \%)$ & $5(10 \%)$ & - \\
$3^{\circ}$ Ciclo (ensino fundamental II) & $94(93,1 \%)$ & $44(88 \%)$ & $50(100 \%)$ \\
Secundário (ensino médio) & $1(1 \%)$ & $1(2 \%)$ & - \\
Escolaridade das mães & & & \\
$1^{\circ}$ Ciclo (ensino fundamental I) & $19(19 \%)$ & $17(34 \%)$ & $2(4 \%)$ \\
$3^{\circ}$ Ciclo (ensino fundamental II) & $61(61 \%)$ & $29(58 \%)$ & $32(64 \%)$ \\
Secundário (ensino médio) & $12(12 \%)$ & $2(4 \%)$ & $6(12 \%)$ \\
Licenciatura (ensino superior) & $8(8 \%)$ & $2(4 \%)$ & \\
\hline
\end{tabular}

Analisando por tipo de violência (Tabela 2), verificamos que dos 18 filhos de mulheres que sofreram apenas violência psicológica, $8(44,4 \%)$ eram do género mascu- lino e 10 do género feminino (55,6\%). Dos 32 filhos de vítimas de violência física e psicológica, $17(53,1 \%)$ são do género masculino e $15(46,9 \%)$ do género feminino.

Tabela 2

Descrição da Amostra por Tipo de Violência

\begin{tabular}{lcccc}
\hline & \multicolumn{2}{c}{ Violência Psicológica } & \multicolumn{2}{c}{ Violência Física e Psicológica } \\
\cline { 2 - 5 } & $N$ & $\%$ & $N$ & $\%$ \\
\hline Género & & & & \\
$\quad$ Masculino & 8 & 44,4 & 17 & 53,1 \\
Feminino & 10 & 55,6 & 15 & 46,9 \\
Ano de Escolaridadedos filhos & & & & \\
$1^{\circ}$ Ciclo (ensino fundamental I) & 2 & 11.1 & 3 & 9,4 \\
$3^{\circ}$ Ciclo (ensino fundamental II) & 16 & 88,9 & 28 & 87,5 \\
Secundário (ensino médio) & 0 & 0 & 1 & 3,1 \\
Habilitações da Mãe & & & & \\
$1^{\circ}$ Ciclo (ensino fundamental I) & 3 & 16,7 & 14 & 43,8 \\
$3^{\circ}$ Ciclo (ensino fundamental II) & 13 & 72,2 & 16 & 50,0 \\
Secundário (ensino médio) & 2 & 11,1 & 0 & 0 \\
Licenciatura & 0 & 0 & 2 & 6,3 \\
\hline
\end{tabular}


Na recolha dos dados, as mulheres incluídas neste estudo foram questionadas se a violência que sofriam era extensível também aos seus filhos (por exemplo, no caso de violência física, se o parceiro também agredia fisicamente os filhos). Com o decorrer das entrevistas foi evidente que diferentes mulheres respondiam de acordo com os diferentes conceitos que tinham acerca de que comportamentos (quer do parceiro quer delas próprias) eram abusivos. Pela impossibilidade de controlar esta variável, optou-se por não a incluir no estudo.

\section{Instrumentos}

Para avaliar a auto-regulação da aprendizagem utilizámos o Inventário de Processos de Auto-Regulação da Aprendizagem - Revisto (IPAAr; Rosário, 2004), composto por nove itens que representam as três fases do processo de auto-regulação da aprendizagem: a planificação; a execução e a avaliação (idem), de resposta tipo Likert com 5 possibilidades (de 1 - Nunca; a 5 - Sempre). O instrumento apresenta características psicométricas adequadas com coeficientes de alpha de Cronbach de 0,84, 0,77 e 0,85 nas escalas Planificação, Execução, Avaliação (Lourenço, 2008).

\section{Procedimentos}

Solicitámos a colaboração formal a equipas de projectos comunitários de apoio a vítimas de violência doméstica contra mulheres, da zona norte de Portugal, tendo sido explicado a finalidade do estudo e a sua operacionalização. Duas técnicas destes programas, procederam ao contacto presencial (nas instalações do projecto de apoio) com mulheres vítimas de violência doméstica, explicando o objectivo do estudo e pedindo o consentimento para a participação dos filhos no estudo. Depois de obtido o consentimento informado, os filhos das vítimas preencheram o instrumento de avaliação, individualmente e sem a presença da progenitora no gabinete. $\mathrm{O}$ processo foi monitorizado por uma técnica do projecto.

Depois da recolha dos dados no grupo das vítimas, e a partir do contacto profundo que as técnicas dos projectos tinham das comunidades, seleccionou-se um grupo de mulheres (com perfil sócio-económico equivalente aos das vítimas). Apenas foram incluídas as mulheres e os filhos que confirmaram nunca terem sido alvo de qualquer tipo de violência doméstica contra mulheres. A recolha dos dados junto dos filhos das vítimas foi feita nas respectivas escolas. Os dados do IPAAr foram tratados com o programa informático do Statistical Package for the Social Sciences (SPSS) para Windows, versão 15.0. As diferenças de médias entre grupos foram realizadas através do teste $t$ de Student e do teste não paramétrico Mann-Whitney.

\section{Resultados}

Os resultados revelam a não existência de diferenças estatisticamente significativas entre os dois grupos (não diferenciando o grupo experimental por tipo de violência), nem no que se refere ao valor total do $\operatorname{IPAAr}(t=191$; $p=0,423)$ nem em nenhum das sub-escalas: Planificação $(t=0,229 ; p=0,448)$, Execução $(t=0,029 ; p=0,133) \mathrm{e}$ Avaliação $(t=0,009 ; p=0,242$; Tabela 3$)$. Analisando a partir do tipo de violência sofrida (Tabela 4) verificamos que os filhos de mulheres que não foram vítimas de violência doméstica apresentam valores superiores, e estatisticamente significativos, na Execução $(M=9,98 ; D P=2,21$; $U=575,5 ; p=0,031)$ e na Avaliação $(M=10,62 ; D P=2,06$; $U=552,0 ; p=0,017)$ quando comparados com os filhos de vítimas simultaneamente de abuso psicológico e físico (Execução: $M=8,78 ; D P=2,85 ;$ Avaliação=9,12; $D P=2,86$ ).

Ao nível das médias de notas obtidas pelos participantes verificamos que os filhos de não-vítimas apresentam uma média significativamente superior $(M=3,83 ; D P=0,55)$ quando comparados com os filhos das vítimas $(M=2,91$; $D P=0,44 ; t=-9,218 ; p=0,000)$. Analisando por tipo de violência, os resultados seguem a mesma tendência, com os filhos de vítimas de abuso psicológico ( $M=2,93 ; D P=0,41$; $t=85,00 ; p=0,000)$ e vítimas simultaneamente de abuso psicológico e físico $(M=2,91 ; D P=0,46 ; t=162,00 ; p=0,000)$ a apresentarem uma média inferior.

Tabela 3

Média, Desvio Padrão e Valor do Teste t de Studant para o IPAAr e Subescalas

\begin{tabular}{lcccccc}
\hline & \multicolumn{2}{l}{ Filhos de Vítimas $(N=50)$} & \multicolumn{2}{c}{ Filhos de Não Vítimas $(N=50)$} & $t$ & $p$ \\
\cline { 2 - 7 } & \multicolumn{1}{c}{$\mathrm{M}$} & $\mathrm{DP}$ & $\mathrm{M}$ & $\mathrm{DP}$ & & \\
\hline Planificação & 9,88 & 2,076 & 9,54 & 2,375 & 0,229 & 0,448 \\
Execução & 9,22 & 2,772 & 9,98 & 2,208 & 0,029 & 0,133 \\
Avaliação & 10,04 & 2,806 & 10,62 & 2,059 & 0,009 & 0,242 \\
Total & 29,14 & 6,596 & 30,14 & 5,803 & 0,191 & 0,423 \\
\hline
\end{tabular}

$p<0,05$. 
Tabela 4

Média, Desvio Padrão e Teste de Diferença de Médias das Medidas de Auto-Regulação, de Filhos de Vitimas de Violência Doméstica Contra Mulheres e Filhos de Não-Vítimas

\begin{tabular}{|c|c|c|c|c|c|c|}
\hline & \multicolumn{2}{|c|}{$\begin{array}{l}\text { Filhos de Vítimas } \\
\qquad(N=50)\end{array}$} & \multicolumn{2}{|c|}{$\begin{array}{l}\text { Filhos de Não Vítimas } \\
\qquad(N=50)\end{array}$} & \multirow[b]{2}{*}{$U$} & \multirow[b]{2}{*}{$p$} \\
\hline & $M$ & $D P$ & $M$ & $D P$ & & \\
\hline \multicolumn{7}{|c|}{ Violência Psicológica $(N=18)$} \\
\hline Planificação & 10,5 & 2,18 & 9,54 & 2,37 & 352,5 & 0,170 \\
\hline Execução & 10,0 & 2,52 & 9,98 & 2,21 & 428,5 & 0,762 \\
\hline Avaliação & 11,67 & 1,85 & 10,62 & 2,06 & 316,0 & 0,059 \\
\hline Total & 32,17 & 5,64 & 30,14 & 5,80 & 345,5 & 0,124 \\
\hline \multicolumn{7}{|c|}{ Violência Psicológica e Física $(N=32)$} \\
\hline Planificação & 9,53 & 1,97 & 9,54 & 2,37 & 797,0 & 0,977 \\
\hline Execução & 8,78 & 2,85 & 9,98 & 2,21 & 575,5 & 0,031 \\
\hline Avaliação & 9,12 & 2,86 & 10,62 & 2,06 & 552,0 & $\mathbf{0 , 0 1 7}$ \\
\hline Total & 27,44 & 6,56 & 30,14 & 5,80 & 601,5 & 0,059 \\
\hline
\end{tabular}

Nota. $U=$ Teste Mann-Whitney.

\section{Discussão}

Os resultados deste estudo revelaram que, apesar de seleccionadas com base num perfil sócio-económico similar, as mulheres vítimas apresentaram habilitações inferiores às não vítimas: bastante mais mulheres com apenas o $1^{\circ}$ ciclo / ensino fundamental (17 vítimas e 2 não vítimas) e bastante menos mulheres tinham um nível de habilitações de secundário/ensino médio (apenas 2 vitimas e 10 não vítimas), o que é congruente com as evidências da investigação (Cunradi, Caetano, \& Schafer, 2002; Renner \& Whitney, 2010). A mesma tendência foi encontrada ao nível da escolaridade dos filhos das vítimas. Todos os filhos das não vítimas frequentavam o ensino fundamental (o nível esperado para as idade dos indivíduos), ao passo que no grupo de filhos de vítimas, $5(10 \%)$ frequentavam o nível inferior (o $1^{\circ}$ ciclo/ensino fundamental I) ao esperado para a idade. Para além disso, os filhos de vítimas registaram uma média de rendimento académico actual inferior aos filhos de não vítimas. Estes dados revelam a existência de perturbação ao nível das trajectórias académicas em relação ao esperado para a idade no grupo dos filhos das vítimas, o que é congruente com outros estudos que encontraram que os filhos de vítimas de violência doméstica contra mulheres apresentam mais dificuldades académicas do que os filhos de não vítimas (Halford et al., 2001; Noble et al., 2005).

As diferenças encontradas apenas ao nível das dimensões da auto-regulação de Execução e de Avaliação, mas não na dimensão de Planeamento sugerem que as dimensões de Execução e de Avaliação são mais sensíveis à exposição das mães a violência física e psicológica con- tra mulheres do que a dimensão do Planeamento. Quer a dimensão de Execução quer a de Avaliação requerem uma organização mais complexa de cognições, emoções e comportamentos do que a dimensão de Planeamento, já que esta envolve essencialmente processos cognitivos, que são relativamente independentes da sua real implementação. Isto é, um aluno pode conseguir planear determinadas estratégias mas pode não conseguir implementálas e, ainda, pode conseguir planear, implementar mas não avaliar. A Execução e Avaliação concretizam o plano ou esquema cognitivo às características de situações concretas, envolvendo naturalmente o ajustamento de vários processos às mesmas (Conway, 2009; Gestsdóttir \& Lerner, 2008). Existe um conjunto de condições de privação que não permitem à criança o desenvolvimento de mecanismos de regulação dos diferentes processos biopsicossociais (e.g., impulsos, cognições, comportamentos) essenciais a um desenvolvimento harmonioso e adaptativo (Conway, 2009; Holden \& Ritchie, 1991). No caso da violência na família (especialmente a violência física), a activação neuropsicológica é especialmente prevalente devido à contínua exposição a situações de maus-tratos, sofridos ou observados (Jaffee et al., 2007). A insegurança, imprevisibilidade e incontrolabilidade que caracterizam estes ambientes familiares dificultam a existência de condições, como a tolerância, a aceitação incondicional, a valorização positiva, a sensibilidade e/ ou a empatia, fundamentais para o desenvolvimento de mecanismos de regulação dos diferentes processos (Kurtz \& Borkowski, 1987; Vygotsky, 1978). Desta forma, é compreensível que a co-ocorrência de violência física e psicológica tenha um efeito negativo na Execução e Avaliação, mas não no Planeamento e que a exposição 
a apenas violência psicológica não tenha, de acordo com os dados deste estudo, um efeito negativo em nenhuma destas três dimensões. Futuros estudos, preferencialmente longitudinais, deverão clarificar esta questão.

Embora a violência doméstica constitua um factor de risco para o desenvolvimento disruptivo da auto-regulação, existem outros factores individuais, parentais e ambientais de vulnerabilização que medeiam este fenómeno (Edleson \& Gewirtz, 2007; Shucksmith, Hendry, \& Glendinning, 1995). O comprometimento dos mecanismos de auto-regulação influencia negativamente a realização cognitiva, regulação motivacional, afectiva, comportamental e ambiental e, consequentemente, o sucesso educativo (Lourenço, 2007; Rehm \& Rokke, 1988; Rosário, 2001; Schunk, 1994; Shucksmith et al., 1995; Zimmerman, 1989).

O facto de se terem encontrado diferenças entre filhos de vítimas e de não vítimas ao nível do rendimento académico, mas apenas terem sido encontradas diferenças entre o grupo de fillhos de vítimas simultaneamente de violência psicológica e física é compreensível. Por um lado, o rendimento académico é um fenómeno multifactorial, sendo a auto-regulação da aprendizagem apenas um dos factores envolvidos. Por outro lado, a exposição a violência contra mulheres tem efeito negativo em múltiplos factores (por exemplo, no funcionamento $\operatorname{cog}$ nitivo - Noble et al., 2005 - e supressão ambiental do QI - Koenen et al., 2003) também envolvidos no rendimento académico. Futuros estudos deverão explorar os efeitos de mediação e de moderação que outros factores preditores do rendimento académico (ex. características de personalidade) têm na relação entre a exposição a violência doméstica e o rendimento académico.

As diferenças encontradas apenas no grupo de filhos de vítimas simultaneamente de violência psicológica e física (e não no grupo de filhos de vitimas apenas de violência psicológica) suportam a ideia de os efeitos cumulativos e interactivos de factores de risco se verificam também ao nível da auto-regulação da aprendizagem em filhos de vítimas de violência contra mulheres. Isto é, a exposição simultânea a violência doméstica psicológica e física contra mulheres afecta negativamente o funcionamento dos filhos das vítimas, o que não acontece no caso de exposição a apenas violência psicológica. Estes resultados são consistentes com as evidências de que diferentes contextos desenvolvimentais e interpessoais estão associados a diferentes processos de auto-regulação (Deci et al., 1996).

Os resultados deste estudo têm implicações a vários níveis, nomeadamente ao nível das intervenções junto destas populações. Para além de favorecer uma compreensão mais holística do fenómeno (por exemplo, ao nível da interacção e acumulação de múltiplos riscos), estes dados suportam a necessidade de intencionalização de estratégias preventivas que promovam o desenvolvi- mento de factores de proteç̧ão que diminuam a probabilidade da transmissão intergeracional da violência doméstica. Porque as características da (nomeadamente a co-ocorrência de diferentes tipos de abuso) estão associadas à severidade do impacto que a violência tem nas vítimas, especial atenção deve ser dada a populações expostas a múltiplos tipos de violência, nomeadamente ao nível do desenvolvimento de intervenções destinadas à diminuição de factores de risco e potenciação de factores de protecção (Edleson \& Gewirtz, 2007). A compreensão dos processos afectados pela exposição à violência doméstica contra mulheres beneficia da compreensão do impacto que a exposição tem em processos instrumentais, envolvidos nas respostas em múltiplos domínios de funcionamento (Jaffee et al., 2007), contribuindo para a promoção de processos de resiliência envolvidos em diferentes situações de desafio (Pesce, Assis, Santos, \& Oliveira, 2004). Por exemplo, devido ao carácter indissociável dos mecanismos de auto-regulação de outros processos como emoções, cognições, etc., sendo as competências sociais e emocionais importantes factores de protecção para um grande número de condições e sendo as intervenções de promoção dessas competências eficazes (e.g., Moreira, Crusellas, Sá, Gomes, \& Matias, 2010), a promoção das competências sociais e emocionais em filhos de vítimas de violência doméstica assume particular importância já que tem o potencial de contribuir para o ajustamento de indivíduos expostos a violência doméstica (Piotrowski, 2011).

Este estudo apresenta algumas limitações que deverão ser consideradas em futuros estudos. Não foi controlado o número de anos nem a severidade dos maus-tratos a que as vítimas foram expostas. Este estudo utilizou uma metodologia quantitativa. No sentido de favorecer uma melhor compreensão das dinâmicas envolvidas no desenvolvimento da auto-regulação da aprendizagem, estudos qualitativos são igualmente necessários. Metodologias que permitam a exploração da interligação entre vários processos psicológicos e a sua organização são particularmente relevantes nesta população. Por exemplo, a narrativa dos indivíduos tem sido reconhecida como uma forma privilegiada de compreensão integrada dos processos psicológicos (Moreira \& Gonçalves, 2010; Moreira, Gonçalves, \& Matias, 2011). Estudos com amostras maiores são necessários, de forma a garantir uma maior validade externa deste tipo de investigações. Apesar de a entrevista conduzida pelos técnicos quer às vítimas quer às não vítimas ter incluído a questão de se o abuso sofrido pela esposa era extensível aos filhos, apercebemo-nos posteriormente que diferentes mulheres tinham diferentes concepções acerca de que tipo de comportamentos (quer o cônjuge quer dela própria) eram considerados abuso. Por esta razão, optámos por não considerar este dado na análise e na interpretação dos dados. 


\section{Referências}

Bandura, A. (2005). The primacy of self-regulation in health promotion. Applied Psychology: An International Review, 54, 245-254.

Boekaerts, M., Maes, S., \& Karoly, P. (2005). Self-regulation across domains of applied psychology: Is there an emerging consensus? Applied Psychology, 54, 149-154.

Carver, S., \& Scheir, F. (1982). Control theory: A useful conceptual framework for personality-social, clinical and health psychology. Psychological Bulletin, 92, 111-135.

Conway, A. (2009). Neuropshycological basis of self-regulation in children and Youth. Reclaiming Children and Youth, 17, 16-22.

Coohey, C. (2004). Battered mothers who physically abuse their children. Journal of Interpersonal Violence, 9, 943-952.

Cunradi, C. B., Caetano, R., \& Schafer, J. (2002). Socioeconomic predictors of intimate partner violence among white, black, and Hispanic couples in the United States. Journal of Family Violence, 17(4), 377-389. doi: 10.1023/ A: 1020374617328

Deci, E. L., Ryan, R. M., \& Williams, G. C. (1996). Need satisfaction and the self-regulation of learning. Learning and Individual Differences, 8, 165-183.

Edleson, J., \& Gewirtz, A. (2007). Young children's exposure to intimate partner violence: Towards a developmental risk and resilience framework for research and intervention. Springer Science, 22, 151-163.

Edwards, V. J., Holden, G. W., Felitti, V. J., \& Anda, R. F. (2003). Relationship between multiple forms of childhood maltreatment and adult mental health in community respondents: Results from the adverse childhood experiences study. American Journal of Psychiatry, 160, 1453-1460.

Gestsdóttir, G., \& Lerner, R. M. (2008). Positive development in adolescence: The development and role of intentional selfregulation. Human Development, 51, 202-224.

Graham-Bermann, S. A., \& Levendosky, A. A. (1998). Traumatic stress symptoms in children of battered women. Journal of Interpersonal Violence, 13, 111-128.

Gross, J. J. (1998). The emerging field of emotion regulation: An integrative review. Review of General Psychology, 2, 271-299.

Gross, J. J. (2002). Emotion regulation: Affective, cognitive and social consequences. Psychophysiology, 39, 281-291.

Halford, W. K., Sanders, M. R., \& Behrens, C. (2001). Can skills training prevent relationship problems in at-risk couples? Four-year effects of a behavioral relationship education program. Journal of Family Psychology, 15, 750-768.

Hazen, A. L., Connelly, C. D., Kelleher, K. J., Barth, R. P., \& Landsverk, J. A. (2006). Female caregivers' experiences with intimate partner violence and behavior problems in children investigated as victims of maltreatment. Pediatrics, 117, 99-109.

Herrenkohl, T. I., Sousa, C., Tajima, E. A., Herrenkohl, R. C., \& Moylan, C. A. (2008). Intersection of child abuse and children's exposure to domestic violence. Trauma, Violence, \& Abuse, 9, 84-99.

Higgins, D. J. (2004). The importance of degree versus type of maltreatment: A cluster analysis of child abuse types. The Journal of Psychology, 138, 303-324.

Higgins, D. J., \& McCabe, M. P. (2000). Multi-type maltreatment and the long-term adjustment of adults. Child Abuse Review, 9, 6-18.
Higgins, D. J., \& McCabe, M. P. (2001). Multiple forms of child abuse and neglect: Adult retrospective reports. Aggression and Violent Behavior, 6, 547-578.

Hirigoyen, M. (1999). El acoso moral: el maltrato psicológico en la vida cotidiana. Barcelona, España: Paidós Ibérica.

Holden, G. W., \& Ritchie, K. L. (1991). Linking extreme marital discord, child rearing, and child behaviour problems: Evidence from battered women. Child Development, 62, 311-327.

Howard, D. E., Feigelman, S., Li, X., Cross, S., \& Rachuba, L. (2002). The relationship among violence victimization, witnessing violence, youth distress. Journal of Adolescent Health, 31, 455-462.

Jaffee, S. R., Caspi, A., Moffitt, T. E., Polo-Tomás, M., \& Taylor, A. (2007). Individual, family and neighborhood factors distinguish resilient from non-resilient maltreated children: A cumulative stressors model. Child Abuse \& Neglect, 31, 231-253.

Jaffee, S. R., Moffitt, T. E., Caspi, A., Taylor, A., \& Arseneault, L. (2002). Influence of adult domestic violence on children's internalizing and externalizing problems: An environmentally informative twin study. Journal of the American Academy of Child and Adolescent Psychiatry, 41, 1095-1103.

Kanfer, H., \& Karoly, P. (1972). Self-control: A behavioristic excursion into the lion's den. Behavior Therapy, 3, 398-416.

Karreman, A., Tuijl, C., Aken, M., \& Dekovic, M. (2006). Parenting and self-regulation in preschoolers: A metaanalysis. Infant and Child Development, 6, 561-579.

Kitzmann, K. M., Gaylord, N. K., Holt, A. R., \& Kenny, E. D. (2003). Child witnesses to domestic violence: A meta- analytic review. Journal of Consulting and Clinical Psychology, 71, 333-352.

Koenen, C., Moffitt, E., Caspi, A., Taylor, A., \& Purcell, S. (2003). Domestic violence is associated with environmental suppression of IQ in young children. Development and Psychopathology, 15, 297-312.

Kurtz, E., \& Borkowski, G. (1987). Development of strategic skills in impulsive and reflective children: A longitudinal study of metacognition. Journal of Experimental Child Psychology, 43, 129-148.

Lengua, L. J. (2002). The contribution of emotionality and selfregulation to the understanding of children's response to multiple risk. Child Development, 73, 144-161.

Lepisto, S., Luukkaala, T., \& Paavilainen, E. (2011). Witnessing and experiencing domestic violence: A descriptive study of adolescents. Scandinavian Journal of Caring Sciences, 25, 70-80.

Lourenço, A. (2007). Processos auto-regulatórios em alunos do $3^{\circ}$ ciclo do ensino básico: Contributos da auto-eficácia e da instrumentalidade. (Dissertação de Mestrado nãopublicada). Universidade do Minho, Braga, Portugal.

Lourenço, A. A. (2008). Processos auto-regulatórios em alunos do $3^{\circ}$. ciclo do ensino básico: Contributos da autoeficácia e da instrumentalidade. (Tese de Doutorado nãopublicada). Universidade do Minho, Braga, Portugal.

Margolin, G. (1998). Effects of domestic violence on children. In P. K. Trickett \& C. J. Schellenbach (Eds.), Violence against children in the family and the community (pp. 57-102). Washington, DC: American Psychological Association.

Margolin, G., \& Gordis, E. B. (2000). The effect of family and community violence on children. Annual Review of Psychology, 51, 445-479. 
Martinez-Torteya, C., Bogat, G. A., von Eye, A., \& Levendosky, A. A. (2009). Resilience among children exposed to domestic violence: The role of risk and protective factors. Child Development, 2, 562-577.

Masten, A. S., \& Coatsworth, J. D. (1998). The development of competence in favorable and unfavorable environments: Lessons from research on successful children. American Psychologist, 53(2), 205-220. doi: 10.1037/0003-066X. 53.2.205

McGee, R. A., \& Wolfe, D. A. (1991). Psychological maltreatment: Towards an operational definition. Development and Psychopathology, 3, 3-18.

Moreira, P., Crusellas, L., Sá, I., Gomes, P., \& Matias, C. (2010). Evaluation of a manual-based programme for the promotion of social and emotional skills in elementary school children: Results from a 4 year study in Portugal. Health Promotion International, 25, 309-317.

Moreira, P., \& Gonçalves, O. F. (2010). Therapist's theoretical orientation and patients' narrative production. Rogers, Lazarus, Shostrom and Cathy revisited. International Journal of Psychology and Psychological Therapy, 10, 79-97.

Moreira, P., Gonçalves, O. F., \& Matias, C. (2011). Client's narratives in psychotherapy and therapist's theoretical orientation: An exploratory analysis of Gloria's narratives with Rogers, Ellis and Perls. Journal of Cognitive and Behavioral Psychotherapies, 11(2), 173-190.

Noble, K. G., Tottenham, N., \& Casey, B. J. (2005). Neuroscience perspectives on disparities in school readiness. Future of Children, 15, 71-89.

Ochsner, K. N., \& Gross, J. J. (2005). The cognitive control of emotion. Trends in Cognitive Sciences, 9, 242.

Pesce, R., Assis, S., Santos, N., \& Oliveira R. (2004). Risco e protecção: Em busca de um equilíbrio promotor da resiliência. Psicologia: Teoria e Pesquisa, 20, 135-143.

Piotrowski, C. C. (2011). Patterns of adjustment among siblings exposed to intimate partner violence. Journal of Family Psychology, 25, 19-28.

Rehm, L. P., \& Rokke, P. (1988). Self-management therapies. In K. S. Dobson (Ed.), Handbook of cognitive-behavioral therapies (pp. 136-166). New York: Guilford.

Renner, L. M., \& Whitney, S. D. (2010). Examining symmetry in intimate partner violence among young adults using sociodemographic characteristics. Journal of Family Violence, 25(2), 91-106. doi: 10.1007/s10896-009-9273-0

Rosário, P. (2001). Diferenças processuais na aprendizagem: Avaliação alternativa das estratégias de auto-regulação da aprendizagem. Psicopedagogia, Educação e Cultura, 5 , 87-102.

Rosário, P. (2004). Estudar o estudar: (Des)venturas do Testas. Porto, Portugal: Porto.

Rosário, P., Soares, S., Núñez Perez, J. C., González-Pienda, J. A., \& Rubio, M. (2004). Processos de auto-regulação da aprendizagem e realização escolar no Ensino Básico. Psicologia, Educação e Cultura, 8, 141-157.

Ryan, R. M., \& Deci, E. L. (2000). Self-determination theory and the facilitation of instrinsic motivation, social development, and well-being. American Psychologist, 55, 68-78.

Schunk, D. H. (1994). Self-regulation of self-efficacy and attributions in academic settings. In D. H. Schunk \& B. J. Zimmerman (Eds.), Self-regulation of learning and performance: Issues and educational applications (pp. 75-99). Hillsdale, NJ: Erlbaum.
Shonkoff, J., \& Phillips, D. A. (Eds.). (2000). From neurons to neighborhoods: The science of early childhood development. Washington, DC: National Academy Press.

Shucksmith, J., Hendry, L., \& Glendining, A. (1995). Models of parenting: Implications for adolescent well-being within different types of family contexts. Journal of Adolescence, $18,253-270$

Silva, A., Duarte, A. M., Sá, I., \& Simão, A. (2004). Aprendizagem autoregulada pelo estudante: Perspectivas psicológicas e educacionais. Porto, Portugal: Porto.

Stover, C. S., \& Berkowitz, S. (2005). Assessing violence exposure and trauma symptoms in young children: A critical review of measures. Journal of Traumatic Stress, 18 , 707-717.

Vermeiren, R., Schwab-Stone, M., Deboutte, D., Leckman, P. E., \& Ruchkin, V. (2003). Violence exposure and substance use in adolescents: Findings from three countries. Pediatrics, $111,535-540$.

Vygotsky, L. S. (1978). Mind in society - The development of higher psychological processes. Cambridge MA: Harvard University Press.

Wolfe, D. A., \& McGee, R. (1994). Dimensions of child maltreatment and their relationships to adolescent adjustment. Development and Psychopathology, 6, 165-181.

Wolfe, D., Crooks, C., Lee, V., McIntyre-Smith, A., \& Jaffe, P. (2003). The effects of children's exposure to domestic violence: A meta-analysis and critique. Clinical Child and Family Psychology Review, 6(3), 171-188

Zimmerman, B. J. (1989). A social cognitive view of selfregulated academic learning. Journal of Educational Psychology, 81(3), 329-339.

Zimmerman, B. J. (1998). Academic studying and the development of personal skill: A self-regulatory perspective. Educational Psychologist, 33, 73-86.

Zimmerman, B. J. (2000). Attaining self-regulation. A social cognitive perspective. In M. Boekaerts, P. Pintrich, \& M. Zeidner (Eds.), Hanbook of self-regulation (pp. 13-39). San Diego, NY: Academic Press.

Zimmerman, B. J., \& Martinez-Pons, M. (1990). Student differences in self-regulated learning: Relating grade, sex and giftedness to self-efficacy and strategy use. Journal of Educational Psychology, 82, 51-59.

Zimmerman, B. J., \& Schunk, D. H. (Eds.). (2001). Selfregulated learning and academic achievement: Theoretical perspectives $\left(2^{\text {nd }}\right.$ ed.). Mahwah, NJ: Erlbaum.
Recebido: 03/05/2011

$1^{a}$ revisão: 03/08/2011

$2^{a}$ revisão: 09/09/2011 Aceite final: 13/09/2011 